\title{
Helium Ion Microscopy for the Imaging of Organic Matrix and Mineral Phase in Developing Tooth Enamel
}

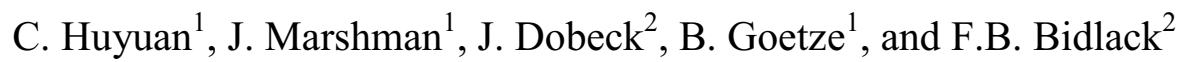 \\ ${ }^{1}$ Carl Zeiss Microscopy, LLC, Ion Microscopy Innovation Center, Peabody, MA 01960, USA \\ ${ }^{2}$ Department of Mineralized Tissue Biology, Forsyth Institute, Cambridge, MA 02142, USA
}

Mammalian tooth enamel is composed mostly of carbonated hydroxyapatite crystals in hierarchical organization. Although during the initial stage of enamel formation the crystals comprise only $30 \mathrm{wt} \%$ in the extracellular matrix, most of the organic material is removed during enamel formation and the end product is comprised of more than $95 \mathrm{wt} \%$ mineral, about $1 \mathrm{wt} \%$ organic material, and residual water. When fully mineralized, tooth enamel consists of extremely long needle shaped crystals, about $70 \mathrm{~nm}$ wide and $30 \mathrm{~nm}$ thick that are organized into bundles (prisms) of a few micrometers in diameter. The delicate interactions between enamel forming cells (ameloblasts), matrix proteins and mineral phase are critical for this exquisitely ordered arrangement [1].

The small size of forming crystals in developing tooth enamel requires the analysis by electron microscopy techniques, whereas the organic matrix is frequently studied using light microscopy of demineralized specimens, as well as immuno-gold labeled sections analyzed with transmission electron microscopy. Although the high-resolution imaging that is possible with field-emission scanning electron microscopy (FE-SEM) has been successfully applied to elucidate mechanisms of bone mineral formation in relation to organic matrix [2], the protein-mineral interactions in tooth enamel have not been demonstrated in this way.

The purpose of this study was to analyze simultaneously both organic and mineral phase in developing tooth enamel. The continuously growing rodent incisors comprise all stages of enamel development from matrix secretion and initial mineral deposition to crystal bundle formation and removal of the organic matrix. Mouse teeth were embedded in epoxy and polished to expose a longitudinal plane through the early stages of enamel development. The major matrix protein amelogenin was identified using 10nm immuno-gold labeling the full-length amelogenin. Electron microscopy was performed on a Zeiss Evo SEM, a Zeiss Merlin FE-SEM and Zeiss Ultra Plus FE-SEM. In addition, an Zeiss Orion ${ }^{\circledR}$ helium ion microscope (HIM) was used. The high-resolution and outstanding depth of field that can be achieved for the imaging of uncoated organic material makes HIM especially suitable to study the spatial organization of organic structures $[3,4]$.

The results illustrate the spatial arrangement of assemblies of the full-length amelogenin in situ upon secretion by ameloblast and before the matrix protein is cleaved (Figure 1). Our findings also reveal the parallel arrangement of elongated protein-assemblies of the major enamel matrix protein amelogenin and show changes of this arrangement with increasing distance from the site of secretion. Furthermore, in later enamel development, the enamel matrix organization in direct vicinity to crystal bundles appears smooth and lacy (Figure 2), whereas in the area between crystal bundles denser and more nodular structures are observed. In conclusion, this approach offers a powerful approach to study samples composed of organic and mineral phase and provides new insights into protein guided mineralization as it allows us to visualize the spatial arrangement of both organic matrix components and mineral phase with few nanometer resolution [5]. 
[1] JP Simmer, et al., Int J Oral Sci, 4(3) (2012), 129.

[2] J Mahamid, et al., Proc Natl Acad Sci U S A, 107(14) (2010), 6316.

[3] WS Vanden Berg-Foels, et al., J Microsc, 246(2) (2012), 168.

[4] SA Boden, et al., Scanning, 34(2) (2012), 107.

[5] The authors acknowledge funding from NIH (grant 1R03DE021765-01A1)

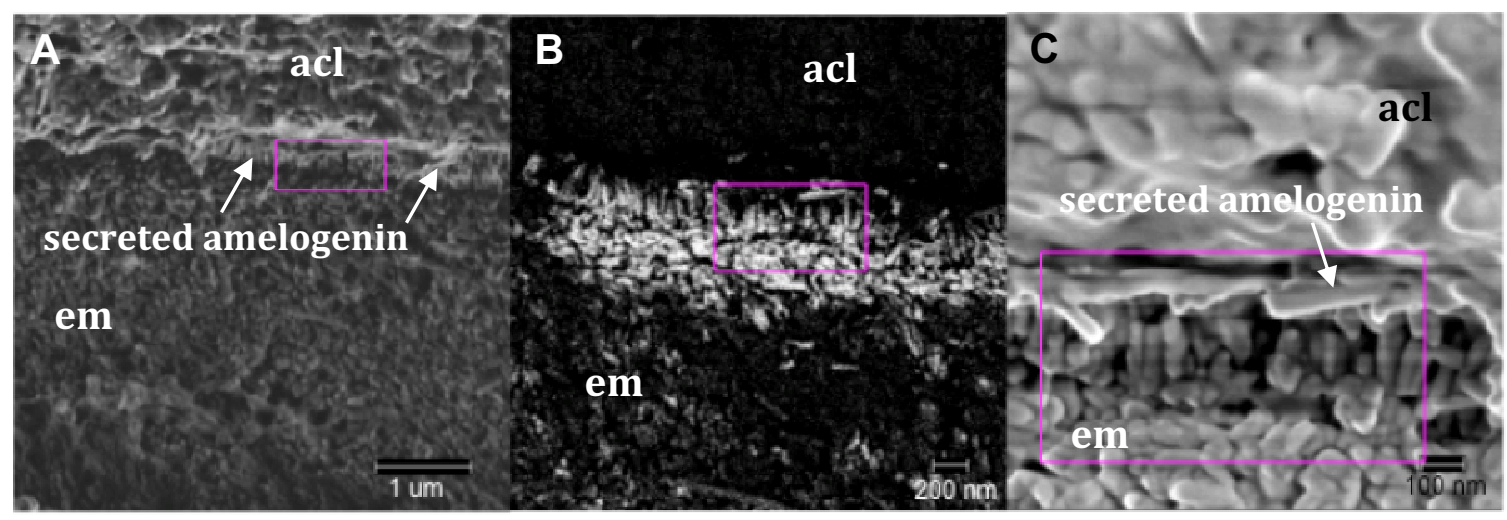

Fig. 1: FE-SEM images of developing mouse incisor enamel in the secretory stage showing the organization of secreted proteins. A) Overview of secretory stage tooth enamel. Marked is the area shown in $\mathrm{B}$ ) in higher magnification and backscattered mode with $10 \mathrm{~nm}$ immunogold labeling on the full-length amelogenin. The area marked is shown in higher magnification in $\mathrm{C}$ ) imaged using secondary electron mode and the in-lens detector.

acl: Ameloblast cell layer; fla: full-length amelogenin; em: enamel matrix.

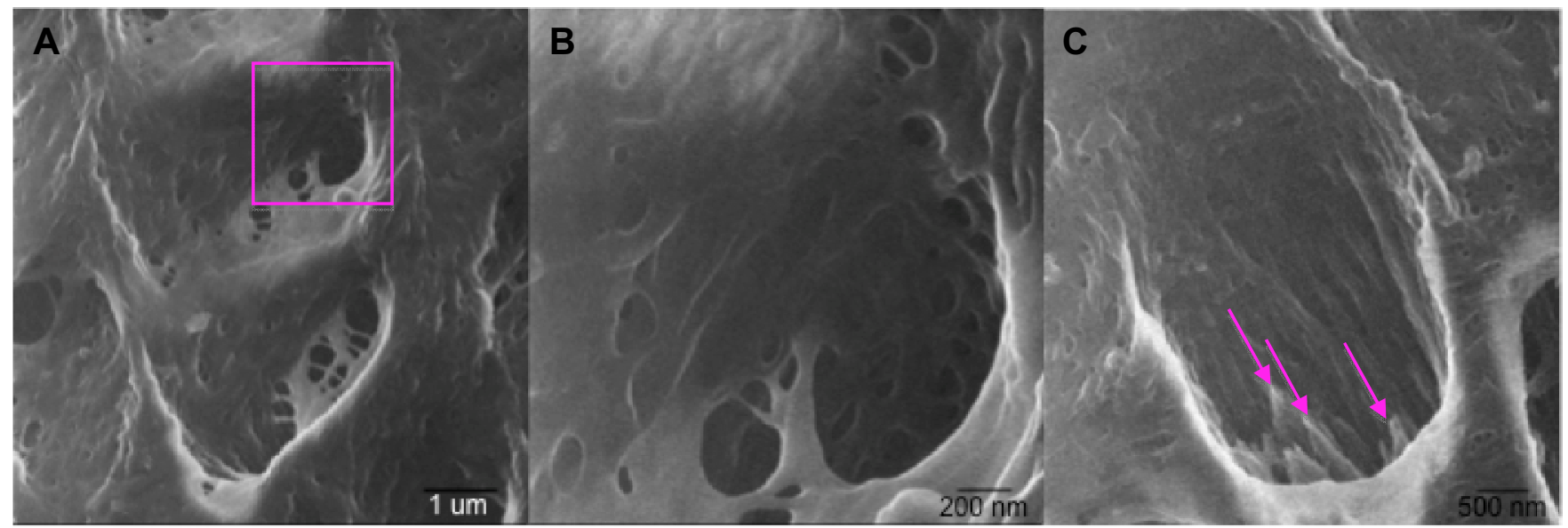

Fig. 2: Helium ion microscope (HIM) images of a developing mouse tooth after 12 second etching in $0.1 \mathrm{M}$ phosphoric acid. A) Removal of the mineral phase leaves empty spaces where forming crystal bundles were located. Area marked is shown in higher magnification in B) demonstrating the high depth of field and resolution revealing the organic matrix organization. C) Later stage of enamel development with crystals (arrows) remaining in the matrix. 